EPJ Web of Conferences 34, 03001 (2012)

DOI: 10.1051/epjconf/20123403001

(C) Owned by the authors, published by EDP Sciences, 2012

\title{
The early years of Pierre: importance of his work for Astrophysics and other fields of Physics
}

\author{
Ronald McCarroll ${ }^{1, a}$ \\ Laboratoire de Chimie Physique-Matière et Rayonnement, (UMR 7614 du CNRS) Université Pierre \\ et Marie Curie, 75231-Paris Cedex 05, France
}

\begin{abstract}
A brief review is given of the pioneering work of Pierre during the years 1974-1981, during which time he investigated the state selective nature of electron capture by multiply charged ions under interstellar conditions. The various stages on the development of the methods used are presented: adiabatic representation and potential energy surfaces, non-adiabatic matrix elements, diabatic transformation, extraction of the S-matrix, optimal choice of reaction coordinates. Finally some very recent results on $\mathrm{D}^{+}+\mathrm{H}_{2} \rightarrow$ $\mathrm{H}^{+}+\mathrm{HD}$ ion-molecule reactions inspired by the early work are presented.
\end{abstract}

\section{Introduction}

I first encountered Pierre in the summer of 1974 at the Les Houches Summer School on Atomic and Molecular physics and the Interstellar Matter. At the time only a few interstellar molecules had been observed: a few interstellar lines of $\mathrm{CH}, \mathrm{CH}^{+}, \mathrm{CN}$ in the visible spectrum, some $\mathrm{UV}$ absorption lines from the rotational levels of ground state of $\mathrm{H}_{2}$, and the $\mathrm{OH}$ maser emission in radio astronomy at a wavelength of $18 \mathrm{~cm}$. At the time, most interest focused on the ionized regions of the interstellar gas. But it was clear that with the development of the $\mathrm{mm}$ and sub-mm waveband, interstellar chemistry was on its way to become a new field of interest in atomic and molecular physics. Some of the topics at the summer school gave some idea of the kind of physical processes which were likely to become important.

Pierre's first venture into this unexplored field in the autumn of 1974 was as much by accident as by design. At the summer school, George Field had conjectured that the ion abundance of $\mathrm{C}^{+}$in ionized interstellar regions might be influenced by recombination of $\mathrm{C}^{2+}$ by a charge transfer reaction with neutral $\mathrm{H}$. The idea seemed interesting and I suggested to Pierre that it was worth investigating. Using an adiabatic representation of the $\mathrm{C}^{2+}+\mathrm{H}$ system, he showed that, while this particular reaction was too slow to be of any real consequence [1], many other common ions of $\mathrm{O}, \mathrm{N}, \mathrm{Si}, \mathrm{S}, \ldots$ charge exchange recombination could indeed be very important process in determining the fractional ionic abundance in many ionized nebulae [2], [3].

\section{Development of theoretical methods}

As a general rule, ion-atom reactions involve the transfer of one or more of the valence electrons from the neutral to the ion. At low and thermal energies, the collision system can be described in an adiabatic (Born-Oppenheimer type) representation, which allows for a quasi-separation of the electronic and nuclear motion. The initial state of each reactant and all the possible states of the reaction products are then correlated to an adiabatic state. A knowledge of the potential energy surface (PES) associated

\footnotetext{
a e-mail: ronald.mac_carroll@upmc.fr
}

This is an Open Access article distributed under the terms of the Creative Commons Attribution License 2.0, which permits unrestricted use, distribution, and reproduction in any medium, provided the original work is properly cited. 
with each adiabatic state, then makes it possible to identify all the reaction channels which do not violate any of the conservation laws of Physics. But to obtain a quantitative estimate of the collision cross sections or rate coefficients, it is essential to have also a knowledge of non-adiabatic coupling between adiabatic states. Much of Pierre's activity was devoted to this complex problem.

The first stage of any calculation involving an adiabatic representation of the collision complex is to determine the potential energy surfaces associated with those electronic states, likely to be involved in the reactive process. This is not s simple problem . Most reactions take place via a near degeneracy (avoided crossings) of excited adiabatic states. Furthermore, since the kinetic temperature of interstellar objects (whether ionized or neutral) is very low (less than e few eV), an accurate representation of the interaction long range inter-molecular distance is required. This kind of problem is not adapted to the use of conventional an-initio methods of quantum chemistry. For that reason, Pierre developed a novel model potential method [4], [5], specifically to treat a collision complex of the type $\mathrm{AB}^{q+}$. The first step involved the construction of a model Hamiltonian, capable of reproducing the energy levels of all the dissociation states of all possible reactant and reactive channels to within $0.1 \%$. Such a precision is essential to obtain a correct description of the long range avoided crossings involving both $\mathrm{A}^{q+}+\mathrm{B}$ and $\mathrm{B}^{q+}+\mathrm{A}$ leading to charge transfer at thermal energies. The eigen energies and eigen functions of the model Hamiltonian are calculated using a fully converged basis. In all cases studied, this approach made it possible to describe the correct long range interactions and the non adiabatic coupling terms in the region of the avoided crossings. An illustration of typical ion atom complexes is shown in figure 1.
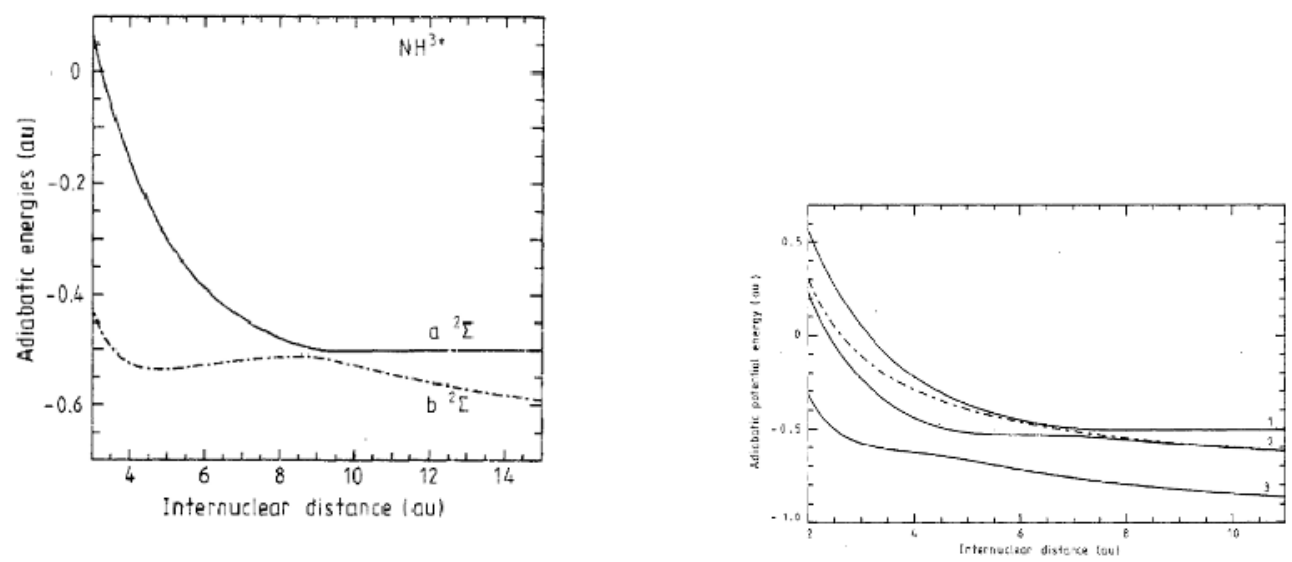

Fig. 1. Illustration of typical avoided crossing in two states and 3-state systems

To obtain collision cross sections and rate coefficients, a knowledge of the non adiabatic couplings is required. These are of two kinds, one due to the radial variation of the adiabatic eigen-functions, the other of rotation (or Coriolis)type arising from the rotation of the molecular axis. In simple 2 state crossings only radial type coupling is involved [6]. In multi-state crossings, both types of couplings can be involved [7]. Examples of typical radial coupling matrix elements are shown in figure 2. However, no discussion of non-adiabatic matrix elements would be complete without some mention of a fundamental defect of an adiabatic representation, which manifests itself in different ways [6],[8]. It is well-known that while the PES are independent of the coordinate system used to describe the collision complex, the non-adiabatic couplings are not. There is no unique way of overcoming the problem, but it can be minimized in such a way by the use of a system of appropriate reaction coordinates which enable the use of a mimimal basis set. Pierre chose to use a reaction coordinate system [9] proposed by Thorson ans Delos [10], which at the time had never been applied to any realistic system. The choice turned out to be an excellent one. 

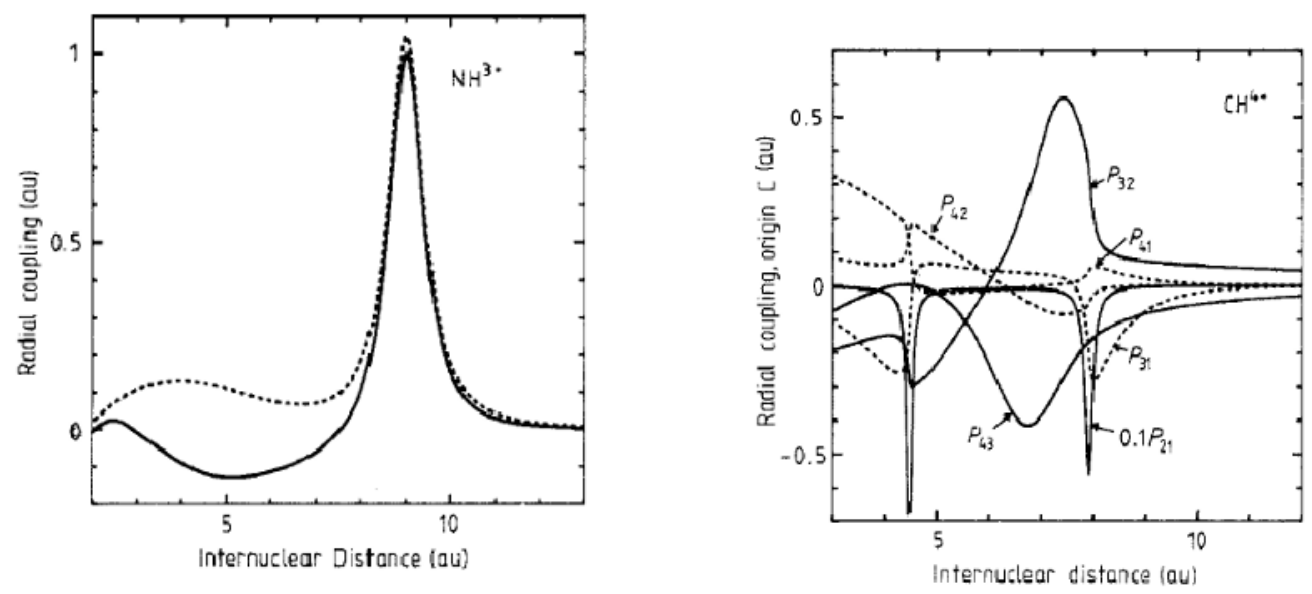

Fig. 2. Typical non-adiabatic matrix elements in a two state $\left(N^{3+}+H\right)$ and a multistate system $C^{4+}+H$

But even with the PES and the non-adiabatic matrix elements, the numerical resolution of the coupled set of differential equations proved to be no mean task. Because the non-adiabatic matrix elements (in whatever coordinate system used) exhibit an uneven structure with sharp peaks in the vicinity if the avoided crossings, it was found necessary to transform to a diabatic basis, in which the differential equations take a simpler form and all of the corresponding matrix elements have a smooth variation with the radial variable. And finally to solve the coupled differential equations, Pierre used a logarithmic derivative approach, which facilitated the way to impose the boundary conditions and facilitated the extraction of the scattering S-matrix.

Over the period from 1974 until 1981, Pierre developed a remarkable ensemble of many new original techniques to treat the dynamics of low energy collisions between ions and atoms. He successfully applied these methods to a variety of different systems and provided the definitive confirmation of the importance of charge transfer recombination processes of doubly and triply charged ions in many ionized nebular interstellar objects. His discovery of the highly state selective nature of charge transfer processes stimulated new experimental activity on energy gain processes in collisions of multiply charged ions with neutral atoms and molecules. The methods he developed have withstood the test of time and are currently still extensively used world-wide.

\section{Applications and some new developments}

Paradoxically, it was only several years after Pierre had left the field to develop new activities, that the importance of his work came to be fully recognized. With the advent of new multiply charged ion sources and of fusion plasma devices, the need for reliable quantitative estimates of state selective electron capture became necessary for diagnostics of the experimental results. As a result, many new applications were carried out when Pierre was no longer directly concerned. But almost all of these applications made use of his methods. To illustrate such an application, a good example (figure 3) is provided by the first detailed experimental results [11], obtained at Oak Ridge National Laboratory. Using a sophisticated merged beam technique enabling the study of low energy collisions. It will be remarked that the theoretical results are in perfect agreement with experiment (see Fig. 3). And in the field of astronomy, another surprising manifestation of state selective charge transfer was discovered in the X-ray spectral line emission from comets due to the radiative decay of highly excited states of highly charged $\mathrm{O}$ and $\mathrm{N}$ ions in the solar wind formed electron capture from the cold cometary gas. 


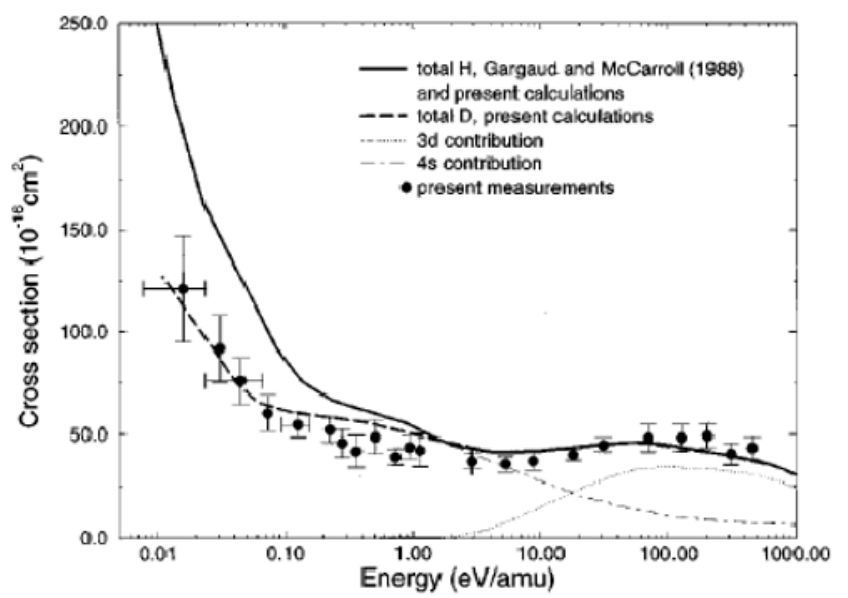

Fig. 3. A comparison of experimental and theoretical results for the electron capture cross section of the $S i^{4}++D$ and $S i^{4}+H$ systems.

Finally, I wish to end with some more recent theoretical developments. The first concerns the choice of reaction coordinates, to which a reference has already been made. One way of tackling the issue is to study the convergence of the basis set. Ideally, a minimal basis set, that is one that includes all states directly involved in the avoided crossing network. But a direct test of the convergence is difficult in practice, since it would involve extending the basis set to include continuum states. A simpler procedure based on Pierre's calculations of the non adiabatic matrix elements has been developed in [12]. In the adiabatic basis, two types of radial coupling matrix elements are involved $A_{i, j}=\langle i|\partial / \partial R| j\rangle$ and $B_{i, j}=\left\langle i\left|\partial^{2} / \partial R^{2}\right| j\right\rangle$. We note that the matrix elements $A_{i, j}$ and $B_{i, j}$ satisfy the relation $d A_{i, j} / d R=$ $D_{i, j}+B_{i, j}$ where $D_{i, j}=\langle\partial i / \partial R \mid \partial j / \partial R\rangle$. If the basis set is complete, the relation $D_{i, j}=\left(\mathbf{A}^{2}\right)_{i, j}$ is fulfilled. This relation is implicitly assumed to be satisfied in practical calculations. But the convergence of the basis set is dependent on the coordinate system. In the Fig. 4, we give an illustration of this dependence in the case of a single avoided crossing. It is seen that the coordinate system adopted by Pierre in his calculations is excellent.

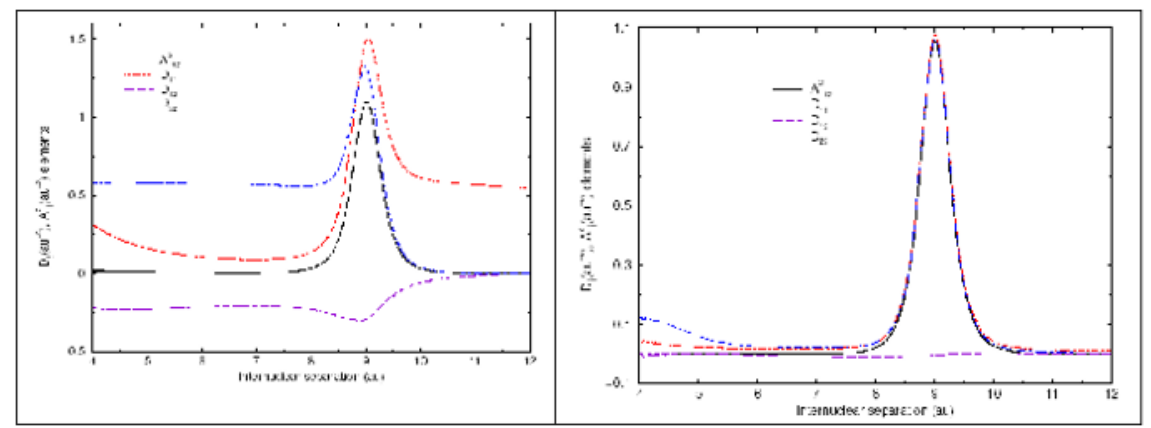

Fig. 4. Illustration of the completeness of the 2 -state basis set for the system $N^{3}++H$. In a complete basis $D_{12}=0, D_{11}=D_{22}=A_{12}^{2}$

A more recent development concerns the reaction of an ion with a rotationally excited molecule. For a reaction with ground state molecules the simple Langevin capture model is satisfactory. But the 


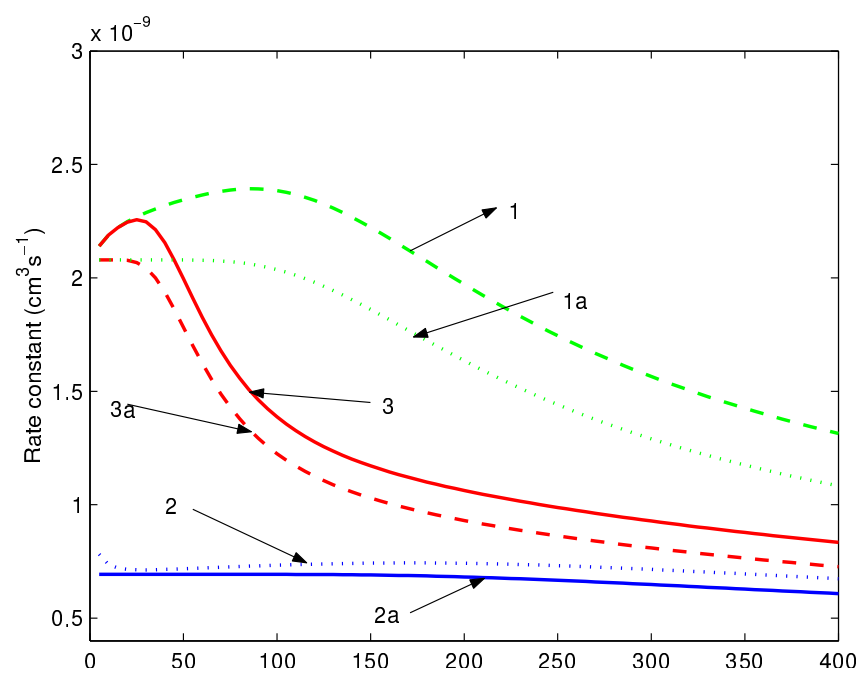

Fig. 5. Rate coefficient of the reaction $\mathrm{D}^{+}+\mathrm{H}_{2} \rightarrow \mathrm{H}^{+}+\mathrm{HD}$

Langevin model in its usual form, is unsatisfactory for reactions with excited molecules. Since rate coefficients involve a thermal average over excited states, it follows that the Langevin model may give increasingly poor results as the temperature increases.

Implicit in the Langevin model is the assumption of statistical mixing of all states of the collision complex leading to energetically accessible reactive channels. But the statistical mixing is subject to two constraints: one concerning the conservation of energy, the other, with the conservation of total angular momentum. This latter constraint is of great importance in reactions with excited molecules. In particular, there can only be statistical mixing of those states of the collision complex a given total angular momentum [13],[14]. Calculations have been made for the rate coefficient for formation of $\mathrm{HD}$ in the $\mathrm{D}^{+}+\mathrm{H}_{2}$ reaction [15]. One set of results in Fig. 5. corresponds to the assumption of a purely radial long range interaction, the other taking account of anisotropic terms. The influence of the anisotropic terms of the potential is appreciable but not of major importance. The agreement with the experimental results is excellent.

\section{Conclusion}

Pierre was a pioneer in the development of methods using an adiabatic representation to study the dynamics of low-energy ion-atom systems. When he started his work, the basic theoretical foundations had been established, but apart from a few simple qualitative deductions based on Landau Zener type models there had been no quantitative applications to interesting physical systems. Pierre remedied this situation. He constructed an ensemble of very efficient computer programs to treat the various aspects of the problem: determination of the relevant PES and of non-adiabatic matrix elements. adiabaticdiabatic transformation, extraction of the scattering matrix. The methods used in his work have been universally adopted and are still commonly applied to-day.

The originality of his work may not always have been fully recognized, in part due to the fact that he himself was not associated with many subsequent applications of his methods from about 1985 onwards. But Pierre had the inner satisfaction of knowing the esteem in which he was held by those who knew him. He impressed us all by his constant dedication to the scientific ideal. And so I would like to say to his surviving wife Odile and to his family just how much Pierre was appreciated by those who were privileged to collaborate with him over the years. 
To the end, Pierre conserved his youthful integrity and enthusiasm. He was always ready to lend a helping hand without any thought of any personal reward. And, let us not forget his remarkable human qualities which made him so highly appreciated by his colleagues and acquaintances.

\section{References}

1. McCarroll, R. and Valiron, P., Astron.E Astrophys, 44, 365 (1976)

2. McCarroll, R. and Valiron, P., Astron.E Astrophys, 53,83 (1976)

3. McCarroll, R. and Valiron, P., J. Physique, 39,C1, 52 (1978)

4. Gayet,R., McCarroll, R. and Valiron, P., Chem. Phys. Lett.,, 58,501(1978)

5. Valiron, P., Gayet,R., McCarroll, R., Masnou-Seeuws, F. and Philippe, M., J. Phys. B: Atom. Molec. Phys., 12,53 (1979)

6. McCarroll, R. and Valiron, P., Astron.E Astrophys, 78,177 (1979)

7. Gargaud, M., Hanssen, J., McCarroll, R. and Valiron, P., J. Phys. B: Atom. Molec. Phys.,, $\mathbf{7 8 1 4}, 2259$ (1981)

8. Opradolce, L., McCarroll, R. and Valiron, P., Astron.E Astrophys 148, 229(1985)

9. Gargaud, M., McCarroll, R. and Valiron, P., J. Phys. B: Atom. Molec. Phys.,, 20,1555 (1987)

10. Thorson, W.R.and Delos, J.B., Phys. Rev. A 18,135 (1978)

11. Pieksma, M., Gargaud, M., McCarroll, R. and Havener, C. C.Phys. Rev. A., 54,R13 (1996)

12. Rabli, D. and McCarroll, R., J. Phys. B: Atom. Molec. opt. Phys., 38,3311 (2005)

13. Pechukas, P. and Light, J.C.,J. Chem Phys.,42, 3281 (1965)

14. McCarroll, R. J. Phys. Chem. A, 113,14845 (2009)

15. McCarroll, R., Book of Invited Talks, XVIII European Conference on Dynamics of Molecular Systems, MOLEC-2010 